\title{
Non-Archimedean Strings and Bruhat-Tits Trees
}

\author{
A. V. Zabrodin \\ Institute of Chemical Physics, SU-117334 Moscow V-334, USSR
}

\begin{abstract}
It is shown that the Bruhat-Tits tree for the $p$-adic linear group $G L(2)$ is a natural non-archimedean analog of the open string world sheet. The boundary of the tree can be identified with the field of $p$-adic numbers. We construct a "lattice" quantum field theory on the Bruhat-Tits tree with a simple local lagrangian and show that it leads to the Freund-Olson amplitudes for emission processes of the particle states from the boundary.
\end{abstract}

\section{Introduction}

In the early days of string theory the string world sheet was thought of as being imbedded into a $D$-dimesnional space-time, with the string action given by the area swept by the world sheet (the Nambu-Goto action [1]). In the course of further development, the approach due to Polyakov [2] proved more fruitful for quantization purposes; the string world sheet was now looked at as an abstract surface with a metric living on it. More precisely, the prescription was to consider a certain two-dimensional theory with its fields taking values in a $D$-dimensional space, and functionally integrate over the fields as well as the metrics. In critical dimension $(D=26)$ the world sheet can be thought of as an algebraic curve over the complex number field, which has allowed for diverse applications for the methods of modern algebraic geometry in perturbative calculations of string amplitudes [3]. Adopting the algebraic-geometrical point of view, it would be quite natural to go beyond the complex number field and to consider other number fields on an equal footing. The first attempts of doing so were made in recent papers [4-6].

A further extension of the notion of the bosonic string world sheet was suggested by Freund and Olson [4], who started with the fact that, as the physically sensible quantities (scattering amplitudes) are given by integrals of complex-valued functions on the world sheet, the world sheet by itself is unobservable. So they chose it to be a manifold over $p$-adic number field $Q_{p}(p-\mathrm{a}$ prime integer). From the conventional point of view based on the archimedean topology, non-archimedean local fields $Q_{p}$ possess a number of peculiar "fractal" 
properties. On the other hand, however, there is a democracy among all the $Q_{p}$ 's and the usual real number field $R$, in that they are all of the completions of the same rational number field $Q$. It may be hoped that "the $p$-adic," or non-archimedean physics may considerably enrich and clarify the conventional "real" physics. In particular, a $p$-adic theory might yield a natural regularization of quantum field theory.

We shall assume that the reader is familiar with basic facts about the $p$-adic fields: the metric properties, integration rules etc. All necessary facts can be found in the books $[7,8]$, and in recent physicists' works wherein the $p$-adic abc has been reviewed several times.

Freund and Olson [4] have given a formal recipe for calculating tree bosonic string amplitudes. Their prescription amounts to setting the correlators of $p$-adic vertex operators $V_{\alpha}$ (unknown as yet, actually) equal to

$$
\left\langle\prod_{j=1}^{N} V_{\alpha_{j}}\left(x_{j}\right)\right\rangle=\prod_{i<j}^{N}\left|x_{i}-x_{j}\right|_{p}^{-\alpha_{i} \alpha_{j}}
$$

with $x_{j} \in Q_{p}$ and $\alpha_{j}$ real $\left(\sum \alpha_{j}=0\right)$. Here and below, ||$_{p}$ is the $p$-adic norm. The correlators (1.1) are to be integrated over $Q_{p}$ with the Haar measure $d x_{j}$ for $N-3$ variables $x_{j}$. For example, the $p$-adic Veneziano amplitude reads

$$
A_{p}\left(k_{1}, k_{2}, k_{3}, k_{4}\right)=\int_{Q_{p}} d x|x|_{p}^{k_{1} k_{2}}|1-x|_{p}^{k_{1} k_{3}},
$$

where $k_{i}$ are $D$-dimensional vectors, $k_{i} k_{j}$ denote the corresponding scalar products, and $k_{1}+k_{2}+k_{3}+k_{4}=0$ is understood, together with $k_{i}^{2}=2$.

The above prescription seems to be a promising suggestion, as it leads to the remarkable Freund-Witten product formula $[9,10]$ for the Veneziano amplitudes:

$$
A_{\infty} \prod_{p} A_{p}=1 \text {. }
$$

Here, $A_{\infty}$ denotes the usual Veneziano amplitude and $p$ runs over all prime numbers. Some non-trivial extensions of this formula also exist [11].

A natural question to ask is whether it is possible to obtain the Freund-Olson amplitudes (1.2) within a Polyakov approach? Or, can one construct a field theory on a $p$-adic manifold with correlators given by (1.1)? We will show that, at the tree level of string perturbation theory at least, the answer is in the affirmative, and we will actually construct the required theory.

The archimedean case teaches us that algebraically non-closed local fields play the role of a boundary of an open string world sheet (thus, $R$ is the boundary of the upper half plane $H$ ). The situation in the non-archimedean case turns out to be quite analogous: the field $Q_{p}$ is in fact the boundary of a discrete homogeneous space $T_{p}$, the so-called Bruhat-Tits tree, which is a natural candidate for the interior of the open $p$-adic string world sheet [12].

The Bruhat-Tits tree $T_{p}$ is an infinite graph containing no cycles. Each vertex is a starting point of $p+1$ edges. The boundary of the tree (i.e., the union of all infinitely remote vertices) can be identified with the $p$-adic number field. All necessary properties of the Bruhat-Tits trees are reviewed in Sect. 2. In Sect. 3 we are concerned with the study of functions and operators on the tree. We introduce 
a local Laplacian, acting in the space of functions defined on the tree vertices, which turns out to be analogous to the usual Laplace operator. Viewing the tree as a sort of lattice approximation to the archimedean world sheet (the vertices being identified with lattice sites), the tree Laplacian becomes a finite-difference analog of the usual Laplacian. We shall construct harmonic functions on the tree and, also, solutions to the Dirichlet and Neumann problems.

In Sect. 4 we consider the "lattice" field theory on the tree with a simple quadratic action

$$
S_{p}[\varphi] \sim \sum_{e}\left(\delta_{e} \varphi\right)^{2} .
$$

Here $\delta_{e} \varphi$ is the difference of the values $\varphi$ takes at the endpoints of a given edge $e$, and the sum is going over all edges. Extrema of the action (1.4) are precisely the harmonic functions on the tree. In the theory governed by the action (1.4), we shall find out the amplitudes of tachyon emission from the boundary of the tree. These amplitudes will be seen to coincide with those postulated by Freud and Olson. Similar to the archimedean case, calculating the three bosonic string amplitudes is reduced to solving the Neumann problem in the interior of the world sheet with $\delta$-functional sources on the boundary.

The same $p$-adic tachyon amplitudes are known to arise from another $p$-adic string action [13], namely, the non-local one given by

$$
\tilde{S}_{p}[\varphi] \sim \int_{Q_{p}} d x d y \frac{(\varphi(x)-\varphi(y))^{2}}{|x-y|_{p}^{2}},
$$

where $\varphi(x)$ is a continuous function on $Q_{p}$. This action seems, at first sight at least, to be quite different from the above action (1.4). However, the fact that the FreundOlson amplitudes are reproduced by both points to some relation between the two. This relation is revealed in Appendix C. Once the local action (1.4) has been introduced, the non-local action (1.5) becomes a secondary object which can be derived from the local action by integrating out the interior of the Bruhat-Tits tree in a way very similar to integrating out the interior of the usual unit disc on the complex plane.

In Sect. 5 we discuss some open problems and possible future directions of the $p$-adic string theory.

In Appendix A we show how to calculate some integrals encountered in Sect. 3. Appendix B contains several useful identities; a direct proof of projective invariance of the amplitudes represented as integrals over the boundary of the tree is also given here.

\section{The Bruhat-Tits Trees}

In the archimedean case the field of real numbers $R$ can be considered as the boundary of the open string world sheet. The interior $H$ of this world sheet (the upper half plane) admits a homogeneous space description as $S L(2, R) / S O(2)$ with a natural $S L(2, R)$-invariant hyperbolic metric. In this metric the world sheet is noncompact, but it can be compactified by adding points at infinity (looked at as the boundary). 
It turns out that this somewhat unusual realization of the interior of the world sheet does have a natural $p$-adic generalization which is achieved by replacing $S L(2, R)$ with its $p$-adic counterpart $G L\left(2, Q_{p}\right)$, the group of invertible $2 \times 2$ matrices over $Q_{p}$ and, correspondingly, $S O(2)$ with the maximal compact subgroup of $G L\left(2, Q_{p}\right)$. To be more precise, consider the homogeneous space

$$
T_{p}=P G L\left(2, Q_{p}\right) / P G L\left(2, Z_{p}\right)
$$

We use the standard notation $P G L(2, K)$ for the group of fractional linear transformations of the projective line $P^{1}(K)$ over a ring $K$ (we will deal with the cases $K=Q_{p}$ or $Z_{p}$ in what follows):

$$
P G L(2, K)=G L(2, K) / K^{*},
$$

where $K^{*}$ denotes the multiplicative group of the ring $K$ and $G L(2, K)$ is the group of invertible $2 \times 2$ matrices with entries in $K$. In $(2.1), Z_{p}$ is the ring of $p$-adic integers, i.e., the numbers $x \in Q_{p}$ such that $|x|_{p} \leqq 1$. Thus, $Z_{p}^{*}$ consists of numbers with unit norm. It is known that $G L\left(2, Z_{p}\right)$ is the maximal compact subgroup of $G L\left(2, Q_{p}\right)$ in the $p$-adic topology and all other maximal compact subgroups are conjugate to it [14].

The space $T_{p}(2.1)$, endowed with a natural $G L\left(2, Q_{p}\right)$-invariant metric, is an infinite discrete space called the Bruhat-Tits tree by the mathematicians. It is known to admit several equivalent descriptions $[14,15]$. We shall see that $T_{p}$ is the true non-archimedean analog of the world sheet. This section contains all necessary information about Bruhat-Tits trees; almost all of it is extracted from $[14,15]$.

Besides (2.1), there exists a more explicit description of the Bruhat-Tits tree in terms of lattices in the $p$-adic space $W=Q_{p} \oplus Q_{p}$. By definition, any free $Z_{p}$-submodule of rank two in $W$ is called a lattice. The simplest example of such a lattice is the submodule $Z_{p} \oplus Z_{p} . Z_{p}$-submodules in $W$, though named lattices, have a faint resemblance to the ordinary lattices in euclidean space. For example, any $Z_{p}$-submodule is compact. This is a manifestation of the following "empirical rule": when going over from the archimedean fields to the non-archimedean ones, continuous objects may become discrete, and vice versa.

Two lattices $l_{1}$ and $l_{2}$ are called equivalent if $l_{1}=\lambda \cdot l_{2}$ for some nonzero $\lambda \in Q_{p}$. Consider the set of equivalence classes of lattices in $W$. One can define a distance between the classes $L_{1}$ and $L_{2}$. It is easy to show that one can choose representatives $l_{i} \in L_{i}(i=1,2)$ such that $l_{1} \subset l_{2}$ (i.e. there are no incommensurable lattices in the non-archimedean case). The factor module $l_{2} / l_{1}$ is finite; it has the form

$$
l_{2} / l_{1}=\left(Z_{p} /(p)^{n}\right) \oplus\left(Z_{p} /(p)^{m}\right)
$$

where $m, n$ are some positive integers and $(p) \subset Z_{p}$ denotes the maximal ideal in the ring $Z_{p}$. The following definition of the distance can be shown to be independent of the choice of the representatives:

$$
d\left(L_{1}, L_{2}\right)=|m-n| .
$$

Obviously, $d\left(L_{1}, L_{2}\right) \geqq 0$ and $d\left(L_{1}, L_{2}\right)=0$ iff $L_{1}=L_{2}$. 


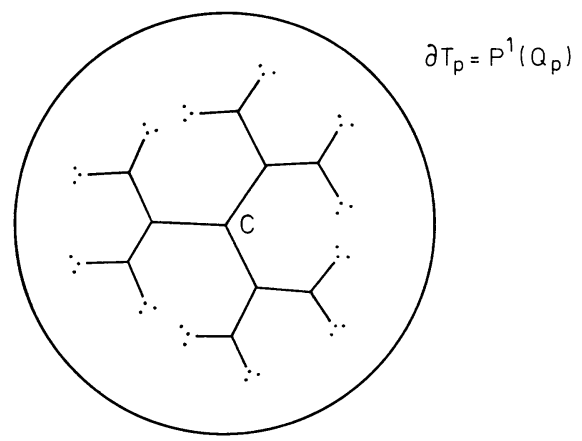

Fig. 1. A Bruhat-Tits tree and its boundary $(p=2)$

The set $T_{p}$ of the lattice equivalence classes with the metric (2.3) is just the Bruhat-Tits tree with the marked point $C$ (the "origin," or the "centre" of the tree) corresponding to the lattice $Z_{p} \oplus Z_{p}$. The definition (2.1) is equivalent to the definition of $T_{p}$ in terms of lattices. The group $G L\left(2, Q_{p}\right)$ acts naturally on the tree leaving the distances invariant, with the maximal compact subgroup $G L\left(2, Z_{p}\right)$ as a stabilizer of the origin $C$.

Now let us describe the tree less formally. The standard way to visualize $T_{p}$ is to draw a graph (or a "lattice" in the usual, non- $p$-adic, sense) which is actually the tree. The vertices of this graph (or, sites of the lattice) correspond to the classes of the $p$-adic lattices in $W$, two vertices (sites) $z_{1}, z_{2}$ being connected by an edge iff $d\left(z_{1}, z_{2}\right)=1$ (Fig. 1), i.e. all nearest neighbours are connected by edges. It is proved in [14] that this graph contains no loops. Each site has exactly $p+1$ nearest neighbours and there is only one path in the tree connecting any two sites.

We have described the "interior" of the tree. Now let us compactify the tree by "adding points at infinity." Any half-infinite path in the tree (without returns) starting at $C$ will be called a ray. The set of all rays will be called the boundary $\partial T_{p}$ of $T_{p}$. Roughly speaking, each ray may be thought of as leading to a point at infinity. The boundary can be naturally identified with the $p$-adic projective line $P^{1}\left(Q_{p}\right)$ [14]. Due to the lack of space we have no possibility to describe this identification in more detail. Finally, there exists an equivalent definition of the boundary in the language of group theory: let $B_{p} \subset G L\left(2, Q_{p}\right)$ be the Borel subgroup, i.e. subgroup of matrices of the form

$$
\left(\begin{array}{ll}
\alpha & \beta \\
0 & \gamma
\end{array}\right),
$$

with $\alpha, \beta, \gamma \in Q_{p}$. Then we have the following isomorphisms:

$$
\partial T_{p} \cong P^{1}\left(Q_{p}\right) \cong G L\left(2, Q_{p}\right) / B_{p} .
$$

It is obvious from these definitions that $G L\left(2, Q_{p}\right)$ [or $\left.P G L\left(2, Q_{p}\right)\right]$ acts on $\partial T_{p}$. In fact, any $g \in P G L\left(2, Q_{p}\right)$ acts on $T_{p}$ by automorphisms. Suppose some ray $x$ (starting at $C$ ) is transformed under the action of $g$ into the half-infinite path $x^{\prime}$ starting at $g(C)$. Strictly speaking, this path is not a ray [since, generally, $g(C) \neq C$ ], but after joining the initial segment $C \rightarrow g(C)$ it becomes the ray which we will denote as $g(x)$. 


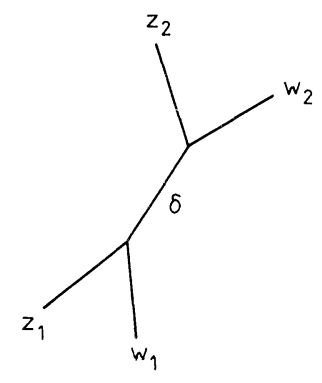

a) $\delta \neq 0$

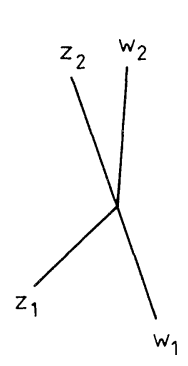

b) $\delta=0$

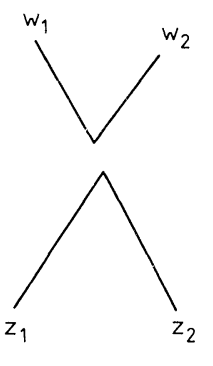

c) $\delta=0$

Fig. 2. Examples of intersections in the tree

For future convenience we need to introduce some more notations. Let $z_{1}, z_{2}$, $w_{1}, w_{2}$ be the vertices of the compactified Bruhat-Tits tree $\bar{T}_{p}=T_{p} \cup \partial T_{p}$ (some of these vertices may lie on the boundary). We shall denote the oriented path connecting the sites $z_{1}, z_{2}$ as $z_{1} \rightarrow z_{2}$. Let

$$
\delta\left(z_{1} \rightarrow z_{2}, w_{1} \rightarrow w_{2}\right)
$$

be the length of the common part of these paths (with the negative sign when the orientations are opposite). So $\delta$ (2.5) is an integer number (Fig. 2). As a rule, in what follows the vertices of $T_{p}$ will be denoted by the letters $z, w$ and boundary points by $x, y$.

There exists a $G L\left(2, Z_{p}\right)$-invariant (i.e., invariant with respect to the "rotations" around $C$ ) distance on the boundary:

$$
|x, y|_{p}=p^{-\delta(C \rightarrow x, C \rightarrow y)} ; \quad x, y \in \partial T_{p} .
$$

Note that for any $x, y \in \partial T_{p},|x, y|_{p} \leqq 1$.

The distance $|x, y|_{p}$ gives rise to the $G L\left(2, Z_{p}\right)$-invariant measure $\mu_{0}$ on the boundary. In order to define it, one needs the notion of a branch in the tree. Let $z, z^{\prime} \in T_{p}$ be nearest neighbours: $d(C, z)=d\left(C, z^{\prime}\right)+1$. Suppose we cut the edge $\left(z, z^{\prime}\right)$, then one of the two subtrees thus obtained does not contain the origin $C$. We call this subtree a branch $B_{z}$ with the origin at $z$ (Fig. 3). The boundary $\partial B_{z}$ of $B_{z}$ is the set of all half-infinite paths (without returns) in $B_{z}$ starting at $z$. The boundary of any branch is an open compact set in $P^{1}\left(Q_{p}\right)$. The measure $\mu_{0}$ is defined by

$$
\mu_{0}\left(\partial B_{z}\right)=p^{-d(C, z)}
$$

Fig. 3. A branch $B_{z}$






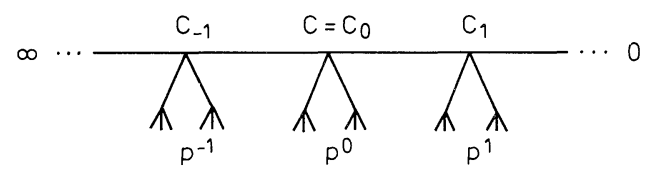

Fig. 4. Identification pattern of the $p$-adic numbers with the points on the boundary of the tree

for any branch $B_{z}$. Its additivity can be easily verified. Locally constant functions, which span a broad class of continuous functions [8] on $P^{1}\left(Q_{p}\right)=\partial T_{p}$, can be integrated over the boundary with this measure.

Up to now we have not introduced any coordinates on $P^{1}\left(Q_{p}\right)$. One may wish to deal with a coordinate function on $P^{1}\left(Q_{p}\right)$, i.e. to identify the boundary with the field of the $p$-adic numbers. In terms of the tree this amounts to choosing three rays leading to the points which are to be identified with 0,1 , and $\infty$. The rays must have no common edges. In order to clarify the rules of this identification, it is useful to draw the tree in a somewhat different way (see Fig. 4). Writing down the number $x \in Q_{p}$ in the form

$$
\begin{gathered}
x=p^{n} u, \\
u=a_{0}+a_{1} p+a_{2} p+\ldots,
\end{gathered}
$$

where the coefficients $a_{i}$ take values in the residue field $F_{p}$ and $a_{0} \neq 0$, the ray $C \rightarrow x$ that corresponds to (2.8) coincides with the path $\infty \rightarrow 0$ as far as the vertex $C_{n}$ and, further, goes within the corresponding branch. The direction to be chosen at the $i^{\text {th }}$ step is determined by the coefficient $a_{i-1}$ in (2.8). then

The distances on $Q_{p}$ have a nice interpretation in terms of the tree: let $x, y \in Q_{p}$,

$$
|1-x / y|_{p}=p^{-\delta(x \rightarrow 0, y \rightarrow \infty)} .
$$

The measure $d \mu_{0}$ defined by (2.7) is related to the Haar measure $d x$ on $Q_{p}$ by the following easily verified relations:

$$
\begin{array}{cc}
d \mu_{0}(x)=d x, & |x|_{p} \leqq 1 \\
d \mu_{0}(x)=d x /|x|_{p}^{2}, & |x|_{p}>1 .
\end{array}
$$

To conclude this section, we would like to make several remarks. Some properties of the Bruhat-Tits tree are analogous to those of one-dimensional chains (the one-dimensional chain corresponds formally to the case $p=1$ ), but in other respects the tree resembles, rather, an infinite dimensional object. In fact, one can define a dimension $D$ of the regular infinite lattice by the following formula:

$$
D=\lim _{R \rightarrow \infty} \frac{\ln N(R)}{\ln R},
$$

where $N(R)$ is the number of lattice sites inside the ball of radius $R$. It is easy to check that in case of the tree $N(R)$ grows exponentially with $R$ and (2.11) has no finite limit, i.e. $D=\infty$. 
It is interesting that for the tree the power of its boundary is greater than that of the interior: $T_{p}$ is countable, whereas $\partial T_{p}$ has the power of the continuum. This probably points to a non-trivial connection between open and closed strings in the non-archimedean case.

\section{The Laplacian and Harmonic Functions on the Tree}

Let us assign a real or complex number $\varphi(z)$ to each site $z \in T_{p}$. In this case we shall say that $\varphi(z)$ is a function on the tree. In this section we study operators acting in the space of such functions.

In the archimedean case the Laplacian is an $S L(2, R)$-invariant operator on the upper half plane $H$. One can expect that its analog on the tree should be a $G L\left(2, Q_{p}\right)$-invariant operator. Let us recall basic facts about invariant operators on homogeneous spaces [16].

Let $G$ be a real or $p$-adic Lie group, $K_{0}$ its subgroup, and $M=G / K_{0}$ the coset space. The invariant operator $\hat{L}$ is characterized by the property

$$
\hat{L}(\varphi(g z))=(\hat{L} \varphi)(g z)
$$

for any $g \in G$ and any function $\varphi(z)$ on $M$. This operator has the kernel $l\left(z, z^{\prime}\right)$ such that $l\left(g z, g z^{\prime}\right)=l\left(z, z^{\prime}\right)$ :

$$
\hat{L} \varphi(z)=\int_{M} d z^{\prime} l\left(z, z^{\prime}\right) \varphi\left(z^{\prime}\right)
$$

where $d z^{\prime}$ is the invariant measure on $M$.

A function $\varphi$ on $M$ may be considered as a function on $G$ constant along every coset class [in this case it will be denoted as $\tilde{\varphi}(g)]$. Every invariant kernel $l\left(z_{1}, z_{2}\right)$ gives rise to the function $L(g)$ on $G$ such that

$$
L\left(g_{2}^{-1} g_{1}\right)=\widetilde{l}\left(g_{1}, g_{2}\right),
$$

$L(g)$ being bi-invariant with respect to $K_{0}$ :

$$
L\left(k g k^{\prime}\right)=L(g)
$$

for any $k, k^{\prime} \in K_{0}$. Conversely, any $K_{0}$-bi-invariant function $L(g)$ yields an invariant kernel according to (3.3). The action of $\hat{L}$ may be represented as a convolution:

$$
\hat{L} \tilde{\varphi}(g)=(\tilde{\varphi} * L)(g)=\int_{G} d g_{1} \tilde{\varphi}\left(g_{1}\right) L\left(g_{1}^{-1} \cdot g\right),
$$

where $d g$ is the Haar measure on $G$.

We are especially interested in the case $G=P G L\left(2, Q_{p}\right), K_{0}=P G L\left(2, Z_{p}\right)$. The algebra of invariant operators on $T_{p}$ (or, in other words, the algebra of $P G L\left(2, Q_{p}\right.$ )bi-invariant functions on $\operatorname{PGL}\left(2, Q_{p}\right)$ with the multiplication given by the convolution) is called the Hecke algebra in mathematical literature [17]. It is wellknown that the Hecke algebra is commutative and is generated by a constant and the characteristic function of the double class

$$
\operatorname{PGL}\left(2, Z_{p}\right)\left(\begin{array}{ll}
1 & 0 \\
0 & p
\end{array}\right) \operatorname{PGL}\left(2, Z_{p}\right) \text {. }
$$


The invariant operator that corresponds to this function (called the Hecke operator $\hat{t}_{p}$ ) acts on a function $\varphi$ on $T_{p}$ as

$$
\hat{t}_{p} \varphi(z)=\sum_{i=1}^{p+1} \varphi\left(z_{i}\right),
$$

where $z_{i}$ are all the nearest neighbours of $z$. So we see that $\hat{t}_{p}$ is a local operator.

We shall define the Laplacian on the tree by the following formula:

$$
\hat{\Delta}_{p}=\hat{t}_{p}-p-1 \text {, }
$$

i.e.

$$
\hat{\Delta}_{p} \varphi(z)=\sum_{i=1}^{p+1} \varphi\left(z_{i}\right)-(p+1) \varphi(z) .
$$

The constant $-(p+1)$ in (3.6) is necessary for the Laplacian to have a zero mode $\varphi=$ const, as in the archimedean case. Note that under a formal limit $p \rightarrow 1,(3.6)$ becomes a finite-difference analog of the second derivative on a one-dimensional chain.

Now our aim is to find eigenfunctions of the Hecke operator (or the Laplacian). In order to do that, let us introduce an auxiliary function $\varepsilon_{\mu, x}(z)(\mu$ is a complex number) by the formulae

$$
\begin{gathered}
\langle z, x\rangle=\delta(C \rightarrow x, C \rightarrow z)+\delta(z \rightarrow x, C \rightarrow z), \\
\varepsilon_{\mu, x}=p^{\mu\langle z, x\rangle} .
\end{gathered}
$$

The following simple fact is crucial for the subsequent constructions: $\varepsilon_{\mu, z}(z)$ is an eigenfunction of the Hecke operator with the eigenvalue $p^{\mu}+p^{1-\mu}$ :

$$
\hat{t}_{p} \varepsilon_{\mu, x}=\left(p^{\mu}+p^{1-\mu}\right) \varepsilon_{\mu, x}(z) .
$$

The proof is straightforward and will be omitted.

Two remarks in order: 1) In our previous paper [12] $\varepsilon_{\mu, x}(z)$ was defined by using horocycles in the tree. Probably, the present definition (3.9) is somewhat simpler. 2) Our method is very similar to the construction of eigenfunctions of the Laplacian on the euclidean space and the upper half plane [18]. Our function $\varepsilon_{\mu, x}(z)$ (the elementary solution) is an analog of the plane wave in the euclidean space.

For $\mu$ equal to 1 in (3.10) we see that

$$
\varepsilon_{1, x}(z)=p^{\langle z, x\rangle} \equiv P(z, x)
$$

is a solution to the Laplace equation for any $x \in \partial T_{p}$ :

$$
\hat{\Delta}_{p} P(z, x)=0 .
$$

Now we immediately obtain a large class of solutions to the Laplace equation (we shall call these the harmonic functions on the tree): for any locally constant function $f(x)$ on $\partial T_{p}$ let

$$
F(z)=\int_{\delta T_{p}} d \mu_{0}(x) f(x) p^{\langle z, x\rangle},
$$


with the measure $\mu_{0}$ (2.7). Obviously, $\hat{\Delta}_{p} F(z)=0$, i.e. $F(z)$ is a harmonic function. In order to investigate the properties of the integral (3.12) it is sufficient to calculate it when $f(x)$ is the characteristic function of the boundary of an arbitrary branch ${ }^{1}$. This calculation is presented in Appendix A; the result is

$$
F_{w}(z)=\int_{\partial B_{w}} d \mu_{0}(x) P(z, x)= \begin{cases}1+p^{-1}-p^{-d(z, w)-1}, & z \in B_{w} \\ p^{-d(z, w)}, & z \notin B_{w}\end{cases}
$$

Thus we see that $F_{w}(z) \rightarrow 0$ when $z \rightarrow \partial T_{p}$ and $z \notin B_{w}$, and $F_{w}(z) \rightarrow 1+p^{-1}$ when $z \rightarrow \partial T_{p}$ and $z \in B_{w}$.

Now we can solve the Dirichlet problem on the tree, which means to reconstruct the harmonic function on $T_{p}$ from its boundary values. The solution is given by the analog of the classical Poisson formula:

$$
\varphi(z)=\frac{p}{p+1} \underset{\partial T_{p}}{ } d \mu_{0}(x) P(z, x) \varphi(x)
$$

with the Poisson kernel (3.11). It follows from (3.12) and (3.13) that $\varphi(z)$ is harmonic on $T_{p}$ and $\varphi(z) \rightarrow \varphi(x)$ when $z \rightarrow x$. The analogy with the classical boundary problems is in fact even more close.

More precisely, let $D(z, w)$ be a symmetric function of the vertices $z, w$ which is a solution to the equation

$$
\hat{\Delta}_{p} D(z, w)=\dot{\delta}_{z, w}
$$

with the boundary condition

$$
\lim _{z \rightarrow 0 T_{p}} D(z, w)=0 .
$$

In (3.15) $\delta_{z, w}$ is the Kronecker delta. One can prove that $D(z, w)$ is uniquely determined and we shall call it the Dirichlet function on the tree ${ }^{2}$. We claim that

$$
D(z, w)=\left(p^{-1}-p\right)^{-1} p^{-d(z, w)},
$$

since this expression possesses all the required properties.

Let us define the analog of the normal derivative at the boundary of the function $\varphi$ by the formula

$$
\partial_{n}^{(p)} \varphi(x)=\lim _{z \rightarrow x}(\varphi(x)-\varphi(z)) p^{d(C, z)}
$$

whenever the limit exists. It is easy to check that

$$
P(z, x)=\left.\left(p-p^{-1}\right) \partial_{n}^{(p)} D(z, w)\right|_{w=x}
$$

as in the classical theory of boundary problems.

Another important task is to find a function $\varphi$ on the tree such that

$$
\hat{\Delta}_{p} \varphi(z)=\varrho(z), \quad \partial_{n}^{(p)} \varphi(x)=0,
$$

\footnotetext{
${ }^{1}$ I.e. the function equal to 1 on $\partial B_{z}$ and 0 otherwise

${ }^{2}$ In order to simplify notations we shall omit the index $p$ in $D(z, w)$
} 
where $\varrho(z)$ is some function (a source). A solution exists only if

and has the form

$$
\sum_{z \in T_{p}} \varrho(z)=0
$$

where

$$
\varphi(z)=\sum_{w \in T_{p}} N(z, w) \varrho(w),
$$

$$
N(z, w)=\frac{1}{2}(d(z, w)-d(C, z)-d(C, w))
$$

is the "Neumann function" on the tree. The Neumann function is the solution to the equation

$$
\hat{\Delta}_{p} N(z, w)=\delta_{z, w}-\delta_{C, z},
$$

and the condition (3.20) is necessary to cancel out the contribution of the auxiliary source in $C$ [see (3.23)].

For completeness let us write down a solution of the Neumann problem on the tree. The problem is to construct a harmonic function $F(z)$ such that

$$
\hat{\partial}_{n}^{(p)} F(x)=J(x),
$$

where $J(x)$ is some continuous function on the boundary. The Neumann function (3.22) has the following boundary value:

$$
N(z, x)=\lim _{w \rightarrow x} N(z, w)=-\delta(C \rightarrow x, C \rightarrow z) .
$$

Using (3.23), (3.25) one can show that the Neumann problem admits a solution only if

$$
\int_{\partial T_{p}} d \mu_{0}(x) J(x)=0,
$$

and this solution is of the form

$$
F(z)=-(p-1) \int_{\partial T_{p}} d \mu_{0}(x) J(x) N(z, x) .
$$

In Appendix A we describe how to calculate integrals of this kind.

It is noteworthy that the Neumann and Dirichlet functions on the tree are quite different things (roughly speaking, the former is a logarithm of the latter). However, such a situation is not so surprising as it might seem, since the boundary is a more powerful set than the interior (see Sect. 2) and so the boundary condition play the most essential role.

\section{Gaussian Model on the Tree and the p-Adic String Amplitudes}

Now we are ready to introduce the action for the $p$-adic string on the Bruhat-Tits tree. Let $X^{\mu}(z)$ be a mapping from the set of vertices on the tree to a $D$-dimensional euclidean space $(\mu=1,2, \ldots, D)$. We shall consider a model with $D$ scalar fields on the tree governed by the action

$$
S_{p}[X]=\frac{\beta_{p}}{2} \sum_{e}\left(\delta_{e} X^{\mu}\right)^{2},
$$


where $\delta_{e} X^{\mu}$ is the difference

$$
\delta_{e} X^{\mu}=X^{\mu}\left(z_{e}^{\prime}\right)-X^{\mu}\left(z_{e}\right)
$$

$\left(z_{e}\right.$ and $z_{e}^{\prime}$ are the endpoints of the edge $e$ ). The sum in (4.1) runs over all edges. The constant $\beta_{p}$ denotes "a $p$-adic string tension." Throughout this paper the summation over repeated indices is assumed. We shall suppose that some appropriate boundary conditions are chosen so as to make the sum convergent.

One can consider this theory as the Gaussian model of statistical mechanics on the tree, then $S_{p}[X]$ is the energy of the system for fixed values of statistical variables $X^{\mu}(z)$. The partition function and mean values are calculated in the usual manner:

$$
\begin{gathered}
Z^{(p)}=\int D X \exp \left(-S_{p}[X]\right), \\
\langle A(X)\rangle_{p}=\left(Z^{(p)}\right)^{-1} \int D X A(X) \exp \left(-S_{p}[X]\right),
\end{gathered}
$$

where the symbol of functional integration should be interpreted as

$$
\int D X \rightarrow \prod_{z} \prod_{\mu=1}^{D} \int_{-\infty}^{+\infty} d X^{\mu}(z)
$$

For the infinite tree these relations are rather formal since a limiting transition to the infinite number of vertices is required for practical computational purposes. In other words, (4.2) should be supplemented with a prescription restricting the integration region in the infinite dimensional space of functions. Due to the great importance of boundary effects in the tree, the concrete form of the limiting process may alter the answers considerably. However, we shall not discuss these questions in the present paper.

It should be noted that some examples of statistical models on the Cayley trees (or Bethe lattices) have already been known in solid state physics and statistical mechanics [19] (without any reference to the $p$-adic context). The homogeneous Cayley trees differ from the Bruhat-Tits trees in only one respect: the number of nearest neighbours of a vertex in the Cayley tree may be any natural number (not necessarily $p+1$ with a prime $p$ ). It would be interesting to interpret the known results about the statistical models on the Cayley trees starting from the $p$-adic point of view.

Now we are going to find the equations of motion (extremum configurations) in the theory with the action (4.1). For this purpose the sum over the edges should be transformed into that over the sites. Suppose the normal derivatives $\partial_{n}^{(p)} \varphi$ and $\partial_{n}^{(p)} f$ exist for the functions $\varphi(z)$ and $f(z)$. Then using the definitions (2.7), (3.7), and (3.17) one can prove "the Green formula" on the tree:

$$
\sum_{e}\left(\delta_{e} \varphi\right)\left(\delta_{e} f\right)=-\sum_{T_{p}} \varphi(z) \hat{\Delta}_{p} f(z)+(p-1) \int_{\partial T_{p}} d \mu_{0}(x) \varphi(x) \partial_{n}^{(p)} f(x) .
$$

After setting $\varphi=f=X^{\mu}$ and varying with respect to $X^{\mu}$ with fixed boundary conditions, we arrive at the following equation of motion:

$$
\hat{\Delta}_{p} X^{\mu}(z)=0 \text {. }
$$

Thus, the solutions are the harmonic functions, which permits us to use the methods of Sect. 3 to calculate the integrals (4.2). The remaining part of this section deals with the tree $N$-point amplitudes. 
Consider the scattering of $N$ identical scalar particles attached to the boundary of the tree (which corresponds to the open string case). Let $E_{p}^{(R)} \subset T_{p}$ denote those tree vertices which lie on the "sphere" of radius $R:\left\{z \in E_{p}^{(R)} \mid d(C, z)=R\right\}$. As our starting point, we postulate the following natural definition of the $N$-point $p$-adic open string amplitudes:

$$
A_{p}^{(N)}\left(k_{1}, \ldots, k_{N}\right)=\lim _{R \rightarrow \infty} \sum_{\left\{z_{j}\right\} \in E_{p}^{(R)}} \frac{\int D X \exp \left(-S_{p}[x]+i \sum_{j=1}^{N} k_{j}^{\mu} \cdot X^{\mu}\left(z_{j}\right)\right)}{\int D X \exp \left(-S_{p}[X]\right)} .
$$

The sum goes over the positions of $N$ points $z_{j}$ on $E_{p}^{(R)}$. The coordinates $X^{\mu}(z)$ are assumed to satisfy the Neumann boundary conditions:

$$
\partial_{n}^{(p)} X^{\mu}(y)=0, \quad y \in \partial T_{p},
$$

which is what one will have required for an open string.

The routine machinery for calculating the Gaussian integrals (4.6) consists in finding out the solution $X_{\mathrm{cl}}^{\mu}$ to the classical equation of motion obtained by varying the exponent:

$$
\beta_{p} \hat{\Delta}_{p} X_{\mathrm{cl}}^{\mu}(z)=-i \sum_{j=1}^{N} k_{j}^{\mu} \delta_{z, z_{J}}
$$

Obviously, this is the problem (3.19). Integrating out the zero mode yields the factor $\delta\left(\sum_{j=1}^{N} k_{j}^{\mu}\right)$ in (4.6), which enforces the condition of solvability of (3.20):

$$
\sum_{j=1}^{N} k_{j}^{\mu}=0
$$

We shall omit this factor in the following, thus keeping in mind that the momentum conservation (4.9) is satisfied. The solution of (4.8) is

$$
X_{\mathrm{cl}}^{\mu}(z)=-i \beta_{p}^{-1} \sum_{j=1}^{N} k_{j}^{\mu} N\left(z, z_{j}\right)
$$

with $N(z, w)$ being the Neumann function (3.22). Substituting $X_{\mathrm{cl}}^{\mu}$ into (4.6) yields

$$
A_{p}^{(N)}\left(k_{1}, \ldots, k_{N}\right)=\lim _{R \rightarrow \infty} \sum_{\left\{z_{j}\right\} \in E_{p}^{(R)}} \exp \left\{\left(2 \beta_{p}\right)^{-1} \cdot \sum_{i, j=1}^{N} k_{i} k_{j} N\left(z_{i}, z_{j}\right)\right\} .
$$

$k_{i} k_{j}$ stands here for $k_{i}^{\mu} k_{j}^{\mu}$; we are going to suppress the index $\mu$. The sum in the exponent is, obviously,

$$
\sum_{i, j=1}^{N} k_{i} k_{j} N\left(z_{i}, z_{j}\right)=-\sum_{i=1}^{N} k_{i}^{2} d\left(C, z_{i}\right)+2 \sum_{i<j}^{N} k_{i} k_{j} N\left(z_{i}, z_{j}\right) .
$$

The summation over $E_{p}^{(R)}$ can be replaced, when $R \rightarrow \infty$, with an integral according to

$$
\sum_{E_{p}^{(R)}} \rightarrow p^{R} \int_{\delta T_{p}} d \mu_{0}(y)
$$

Note also that when $d\left(C, z_{j}\right) \rightarrow \infty$ it follows from (3.22) that

$$
N\left(z_{i}, z_{j}\right)=-\delta\left(C \rightarrow z_{i}, C \rightarrow z_{j}\right) .
$$


Collecting everything together, we arrive at

$$
\begin{aligned}
& A_{p}^{(N)}\left(k_{1}, \ldots, k_{N}\right)=\lim _{R \rightarrow \infty} p^{N R} \exp \left(-\left(2 \beta_{p}\right)^{-1} k_{i}^{2} N R\right) \\
& \quad \times \int_{\delta T_{p}} \prod_{j=1}^{N} d \mu_{0}\left(y_{j}\right) \exp \left(-\beta_{p}^{-1} \cdot \sum_{i<j}^{N} k_{i} k_{j} \delta\left(C \rightarrow y_{i}, C \rightarrow y_{j}\right)\right) .
\end{aligned}
$$

The limit exists only if

$$
k_{i}^{2}=2 \beta_{p} \ln (p),
$$

which, as will be clear in Appendix B, is at the same time the condition for the projective $G L\left(2, Q_{p}\right)$-invariance of $(4.15)$. Assuming (4.16) to hold, choose the scale so that $k_{i}^{2}=2$, i.e., set $\beta_{p}=(\ln (p))^{-1}$.

Finally, using (2.6), Eq. (4.15) takes the form

$$
A_{p}^{(N)}\left(k_{1}, \ldots, k_{N}\right)=\int_{\partial T_{p}} \prod_{j=1}^{N} d \mu_{0}\left(y_{j}\right) \prod_{i<j}^{N}\left|y_{i}, y_{j}\right|_{p}^{k_{2} k_{j}} .
$$

The group $P G L\left(2, Q_{p}\right)$ acts projectively on $\partial T_{p}=P^{1}\left(Q_{p}\right)$ (see Sect. 2). It is shown in Appendix B that the condition $k_{i}^{2}=2$ ensures the projective invariance of (4.17).

This sort of "gauge" invariance does not lead to an infinite factor, since the integration is confined to a compact region. We can nevertheless fix a gauge in (4.17) so as to be able to compare it with the amplitudes of [4]. This is carried through in the standard way. As a group element is determined by three parameters, it is possible to fix arbitrarily three variables, as, for example, $y_{N}=y_{N}^{0}$, $y_{N-1}=y_{N-1}^{0}, y_{N-2}=y_{N-2}^{0}$. The measure thus is to be replaced with

$$
\prod_{j=1}^{N} d \mu_{0}\left(y_{j}\right) \rightarrow \prod_{k=N-2}^{N} \delta\left(y_{k}, y_{k}^{0}\right) \prod_{N-2 \leqq m<m \leqq N}\left|y_{m}, y_{n}\right|_{p} \prod_{j=1}^{N} d \mu_{0}\left(y_{j}\right),
$$

where $\delta(x, y)$ is the $\delta$-function on $\partial T_{p}$ defined in an obvious way.

To compare (4.17) with the known expressions for the $p$-adic amplitudes we identify the boundary of the tree with $Q_{p}$, as explained in Sect. 2. Then the measure $d \mu_{0}$ undergoes the transformation (2.10), while the distance $|x, y|_{p}$ is transformed as

$$
|x, y|_{p} \rightarrow \begin{cases}|x-y|_{p} ; & |x|_{p} \leqq 1,|y|_{p} \leqq 1 \\ \left|x^{-1}-y^{-1}\right|_{p} ; & |x|_{p} \geqq 1,|y|_{p} \geqq 1 \\ 1, & \text { otherwise }\end{cases}
$$

On the left-hand side $x$ and $y$ denote the points on the boundary, whereas on the right-hand side the images thereof under the identification $\partial T_{p} \cong Q_{p}$, that is, $x$ and $y$ are proper $p$-adic numbers on the right-hand side of (4.19). Thus, once we have fixed a gauge, Eq. (4.17) takes the form of an $(N-3)$-fold integral over $Q_{p}$. Setting, as usual, $y_{N}=0, y_{N-1}=1, y_{N-2}=\infty$ and using (4.18) we see (4.17) to be equal to

$$
A_{p}^{(N)} \sim \int_{Q_{p}} \prod_{j=1}^{N-3} d y_{j} \prod_{i=1}^{N-3}\left|y_{i}\right|_{p}^{k_{i} k_{N}}\left|1-y_{i}\right|_{p}^{k_{2} k_{N}-1} \prod_{m<n}\left|y_{m}-y_{n}\right|_{p}^{k_{m} k_{n}},
$$

which is precisely the Freund-Olson amplitude. 
Note that the expression (4.17) for the amplitudes has some advantages over (4.20), as the integration goes over a compact domain and all the integration variables enter symmetrically.

It is known that $p$-adic $N$-point amplitudes can be calculated explicitly for arbitrary $N[10,20]$, the answer being expressed as a sum of tree diagrams of an effective field theory. Remarkably, these tree graphs can be visualized in terms of the Bruhat-Tits trees.

To complete our discussion of the local action for the $p$-adic string, we wish to point to its relation to the non-local action on $Q_{p}$ proposed in [13]. It turns out that (1.5) is in fact an "effective" action on the boundary of the tree, obtained from (4.1) by integration out the interior of the tree. This is demonstrated in Appendix C, where we present an alternative way to calculate the amplitude (4.6).

\section{Concluding Remarks}

We hope that the above results will be useful in clarifying the physical meaning of non-archimedean field theory. Thus, the Bruhat-Tits tree might be considered as a natural "lattice" approximation to the world sheet and thus used for regularisation purposes. In many respects, the tree seems preferable as compared with the usual triangular or quadrangular lattices.

Finally, we would like to discuss possible future developments of the nonarchimedean strings. An essential remark to the above construction is that it is only partly analogous to the Polyakov approach in the archimedean case, since we have at present no idea of what the integral over metrics may correspond to. The point is that the Bruhat-Tits tree is an analog of the unit disc (or, the upper half plane) with fixed hyperbolic metric, and it is unclear what kind of tree transformations might correspond to changing the metric. The Bruhat-Tits tree actually does have a huge automorphism group [21] [which is much larger than $G L\left(2, Q_{p}\right)$ !] but it is very much unlike the usual conformal group in that it does preserve the distances in the tree. A more hopeful fact is that an analog of the circle diffeomorphism group $\operatorname{Diff}\left(S_{1}\right)$ is known for the tree [22]: the group consists of exactly those automorphisms of the boundary, $\partial T_{p}$, that extend to the interior of the tree to within some neighbourhood of the boundary.

From the adelic point of view it is natural to consider the trees $T_{p}$ for all $p$ simultaneously and to unify them all into the space $\otimes_{p} T_{p}$ whose points correspond, roughly speaking, to rational lattices in a two-dimensional real linear space. Presumably, field-theoretic models on trees can serve, in a sense, as "building blocks" for conformal field theories on the disc (in analogy to the adelic string hypothesis [9]).

It may be conjectured that a physical theory built over non-archimedean fields should have a richer algebraic structure as compared with the usual "real" or "complex" theories, due to the fact that the $Q_{p}$ field has infinitely many algebraic extensions, whereas real numbers, only one. The study of these extensions and their corresponding Galois groups is a subject of an advanced mathematical discipline, the algebraic number theory [23]. The question arises as to how all these extensions may show up in the non-archimedean strings. It had been 
conjectured (by analogy with the archimedean case) that while the field $Q_{p}$ corresponded to the open string, quadratic extensions thereof, $Q_{p}(\sqrt{\tau})$, should correspond to the closed string. It seems that this conjecture has been taken for granted in all subsequent works on $p$-adic strings. However, the true situation is more subtle.

First of all, $Q_{p}$ has three distinct quadratic extensions [8]. Further, even if these were somehow dealt with, the role played by extensions of higher degree remains unclear. (The strings - if any - which might correspond to these extensions have been called "even more closed" in [10].)

As more appropriate point of view, we would like to point to the following possibility: algebraically non-closed local fields (including all finite degree extensions of $Q_{p}$ ) might correspond to different types of open strings.

In fact, let $K$ be an extension of $Q_{p}$ of degree $n\left(\left[K: Q_{p}\right]=n\right)$ with the ramification index $e\left(K / Q_{p}\right) \equiv e[23]$. Set $f=n / e$. A Bruhat-Tits tree $T(K)$ can be assigned to any such $K$ by the following procedure: 1) insert onto each edge of $T_{p}$ $e-1$ new vertices separated by equal distances, 2 ) draw new branches in such a way that each vertex have exactly $p^{f}+1$ nearest neighbours. Clearly, the tree $T(K)$ has a boundary $\partial T(K)$, being therefore the interior of an open string world sheet. The constructions of Sects. 3 and 4 obviously go through when applied to $T(K)$.

It may be expected that the non-archimedean closed string is related to the completion of the algebraic closure of $Q_{p}$, the field $\Omega$, and, as such, should be described in terms of some limiting transition. Even if this is not exactly the case, our results point to a possible highly non-trivial relation between closed and open non-archimedean strings.

A sensible thing to do first would be to investigate in what way distinct finite extensions are related physically. The bosonic string theory is not likely to be of any use in doing so, since it involves all finite extensions independently and on an equal footing. Quite another situation might be encountered in the theory of fermionic $p$-adic strings. Indeed, by a direct analogy with the archimedean case, we can propose the following non-local form of the open fermionic $p$-adic string action over the $p$-adic field:

$$
\tilde{S}_{p}[\psi] \sim \int_{Q_{p}} d x d y \psi(x) \frac{\operatorname{sign}_{\tau}(x-y)}{|x-y|_{p}} \psi(y) .
$$

Here, $\operatorname{sign}_{\tau}(x)$ is the multiplicative unitary character on $Q_{p}^{*}$ that corresponds to the quadratic extension $Q_{p}(\sqrt{\tau})$ [8]. Note that parafermions emerge naturally in the $p$-adic case when one replaces $\operatorname{sign}_{\tau}(x)$ in (5.1) with any multiplicative unitary character $\omega(x)$ on $Q_{p}^{*}$. By the class field theory [23] distinct characters $\omega(x)$ correspond to distinct algebraic extensions of $Q_{p}{ }^{3}$. Thus the fermions on $Q_{p}$ should know about algebraic extensions of $Q_{p}$. This will be considered in more detail in a separate paper [24].

When this work had been finished. J. Nekovar informed me about Cartier's work [25] wherein harmonic functions on the tree had been considered.

\footnotetext{
${ }^{3}$ This is exactly the reason for $R$ to admit precisely one such character, the $\operatorname{sign}(x)$
} 


\section{Appendix A}

In this appendix, we discuss the calculation of the integrals (3.13) and (3.27) over the boundary of the tree. As an example, consider

$$
F_{w}(z)=\int_{\partial B_{w}} d \mu_{0}(x) p^{\langle z, x\rangle}
$$

for arbitrary branch $B_{w}$. Two cases are distinct:

1) $z \in B_{w}$. In this case, as shown in Fig. 5a, the vertices $C, w$, and $z$ lie on the same "line." Let the vertices $z_{k}$ belong to the segment $w \rightarrow z$ of the above "line," with $d\left(C, z_{k}\right)=k(k=d(C, w), d(C, w)+1, \ldots, d(C, z))$. To simplify the notation, we will write $d(z)$ instead of $d(C, z)$. The vertex $z_{k}$ has $p-1$ nearest neighbours $z_{k, i}$ which do not belong to the path $C \rightarrow z$. Consider the branches with the origins at $z_{k, i}$. The boundary of each of these branches has the measure $p^{-k-1}$, while the function $\langle z, x\rangle$ is constant on these boundaries, $\langle z, x\rangle=2 k-d(z)$. Adding up the contributions of these branches, together with that of the branch $B_{z}$, we obtain

$$
F_{w}(z)=\sum_{k=d(w)}^{d(z)-1}(p-1) p^{-k-1} p^{2 k-d(z)}+p^{-d(z)} p^{d(z)}=1+p^{-1}-p^{-d(z)-1} .
$$

2) $z \notin B_{w}$. This case, in turn, involves several possible positions of $C$ relative to $z$ and $w$. We consider in detail the case when the three points do not lie on the same "line" (Fig. 5b). Then

$$
\begin{gathered}
\mu_{0}\left(\partial B_{w}\right)=p^{-d(w)}, \quad\langle z, x\rangle=2 d\left(z_{0}\right)-d(z) ; \\
F_{w}(z)=p^{-d(w)} p^{2 d\left(z_{0}\right)-d(z)}=p^{-d(z, w)} .
\end{gathered}
$$

Other cases can be considered analogously, with the same result (A.3).

The calculation of the integral

$$
\int_{\delta B_{w}} d \mu_{0}(x) N(z, x)
$$

[required in order to check (3.27)] goes through in a similar way.

\section{Appendix B}

In this appendix we give the proof of the projective invariance of the integrand in (4.17).

To begin with, note the following useful identity:

$$
\delta\left(w_{1} \rightarrow w_{2}, z_{1} \rightarrow z_{2}\right)+\delta\left(w_{2} \rightarrow w_{3}, z_{1} \rightarrow z_{2}\right)=\delta\left(w_{1} \rightarrow w_{3}, z_{1} \rightarrow z_{2}\right) .
$$

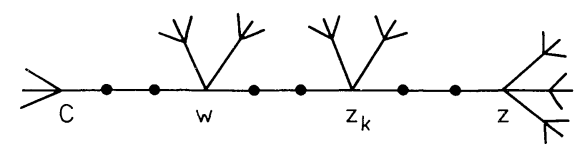

a)

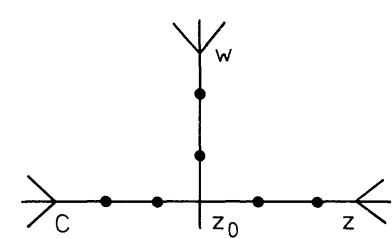

b)

Fig. 5a and b. Different relative positions of the points $C, z, w: \mathbf{a} z \in B_{w}, \mathbf{b} z \notin B_{w}$ 
As noted in the main body of the paper, the elements of $G L\left(2, Q_{p}\right)$ act on $T_{p}$ as graph automorphisms, i.e., they leave the distances (2.3) invariant. Clearly, $G L\left(2, Q_{p}\right)$ acts on $\partial T_{p}$ as well, although distances (2.6) may be changed, due to a shift of the centre $C$. Let $g \in G L\left(2, Q_{p}\right)$. Using the invariance of (2.3) and the identity (B.1), one can prove the following identities:

$$
\begin{gathered}
\langle g(z), g(x)\rangle=\langle z, x\rangle+\langle g(C), g(x)\rangle, \\
\langle z, x\rangle-\left\langle z, x^{\prime}\right\rangle=2 \delta\left(x^{\prime} \rightarrow x, C \rightarrow z\right)
\end{gathered}
$$

for all $z \in T_{p}$ and $x, x^{\prime} \in \partial T_{p}$. For brevity, denote $g^{-1}(C)=C^{\prime}$. Using (B.1)-(B.3), we obtain

$$
2 \delta(C \rightarrow x, C \rightarrow y)-2 \delta\left(C^{\prime} \rightarrow x, C^{\prime} \rightarrow y\right)=\left\langle C^{\prime}, x\right\rangle+\left\langle C^{\prime}, y\right\rangle
$$

whence

$$
\begin{aligned}
|g(x), g(y)|_{p}^{2} & =p^{2 \delta(C \rightarrow x, C \rightarrow y)-2 \delta\left(C^{\prime} \rightarrow x, C^{\prime} \rightarrow y\right)}|x, y|_{p}^{2} \\
& =p^{\left\langle g^{-1}(C), x\right\rangle+\left\langle g^{-1}(C), y\right\rangle}|x, y|_{p}^{2}=P\left(C^{\prime}, x\right) \cdot P\left(C^{\prime}, y\right)|x, y|_{p}^{2}
\end{aligned}
$$

[see (3.11)]. Hence the transformation law for the measure $d \mu_{0}$ is

$$
d \mu_{0}(\tilde{x})=P\left(C^{\prime}, x\right) d \mu_{0}(x)
$$

with $\tilde{x} \equiv g(x)$.

Applying these relations to (4.17) and making use of the mass shell condition $k_{i}^{2}=2$, we arrive at

$$
\prod_{i<j}^{N}\left|\tilde{y}_{i}, \tilde{y}_{j}\right|_{p}^{k_{k} k_{j}}=\prod_{j=1}^{N} P^{-1}\left(C^{\prime}, y_{j}\right) \prod_{i<j}^{N}\left|y_{i}, y_{j}\right|_{p}^{k_{2} k_{j}} .
$$

In view of (B.6), it becomes obvious that the integrand in (4.17) is projectively invariant.

\section{Appendix C}

We are going to demonstrate how the non-local action (1.5) on $Q_{p}$, proposed in [13], can be derived from (4.1) by integrating out the interior of the Bruhat-Tits tree.

Consider the sphere $E_{p}^{(R)}$ of radius $R$ centered at $C$, i.e., (see Sect. 4) the set $\{z \mid d(C, z)=R\}$. Let $J_{R}(z)$ be a source supported by $E_{p}^{(R)}$ :

$$
J_{R}(z)=i \sum_{j=1}^{N} k_{j} \cdot \delta_{z, z_{J}} ; \quad z_{j} \in E_{p}^{(R)},
$$

where $k_{j}$, as usual, are the conserved momenta. Let, further, $X_{R}(z)$ be a function on $E_{p}^{(R)}$. We have the following obvious equality:

$$
\begin{aligned}
& D X \exp \left(-S_{p}[X]+i \cdot \sum_{E_{p}^{(R)}} J_{R}(z) X(z)\right) \\
& \quad=D X_{R} \exp \left(i \sum_{E_{p}^{(R)}} J_{R}(z) X_{R}(z)\right)_{\left.X\right|_{E_{p}} ^{R}=X_{R}} D X \exp \left(-S_{p}[X]\right) .
\end{aligned}
$$


The integrations with respect to $D X$ are understood to be carried out with the Neumann boundary conditions. For finite values of $R$ we have a finitely multiple integral with respect to $D X_{R}$.

The meaning of the transformation (C.2) is that it allows one to carry out first the inner integration on the right-hand side. Shifting the integration variable as

$$
X(z)=X_{0}(z)+X_{q}(z)
$$

where $X_{0}^{(R)}(z)$ is harmonic inside $E_{p}^{(R)}$ [i.e., for $\left.d(C, z) \leqq R-1\right]$ and equals $X_{R}(z)$ at any point of each branch $B_{z}$ with origin at $z \in E_{p}^{(R)}$. It can be shown that $X_{0}^{(R)}$ is defined uniquely. The function $X_{q}(z)$ is zero on $E_{p}^{(R)}$ and otherwise arbitrary. We wish to integrate $X_{q}$ out of the right-hand side of (C.2). Substituting (C.3) into (C.2), the action takes the form

$$
\begin{aligned}
-2 \ln (p) S_{p}[X]= & \sum_{T_{p}} X_{q}(z) \hat{\Delta}_{p} X_{q}(z)+\sum_{T_{p}} X_{0}^{(R)}(z) \hat{\Delta}_{p} X_{q}(z) \\
& +\sum_{T_{p}} X_{q}(z) \hat{\Delta}_{p} X_{0}^{(R)}(z)+\sum_{T_{p}} X_{0}^{(R)}(z) \hat{\Delta}_{p} X_{0}^{(R)}(z) .
\end{aligned}
$$

By the definition of $X_{0}^{(R)}, \hat{\Delta}_{p} X_{0}^{(R)}(z)$ is non-zero only on $E_{p}^{(R)}$, which means that the third term in (C.4) is zero. So is the second term, since, by a straightforward application of Green's formula (4.4), it equals the third term. Thus the action is

$$
-2 \ln (p) S_{p}[X]=\sum_{T_{p}} X_{q} \hat{\Delta}_{p} X_{q}+\sum_{E_{p}^{(R)}} X_{0}^{(R)} \hat{\Delta}_{p} X_{0}^{(R)}
$$

To normalize (C.2), divide it by the same expression with $J_{R}=0$. The resulting ratio will be denoted as $A_{p}\left[J_{R}\right][\operatorname{see}(4.2)]$ :

$$
A_{p}\left[J_{R}\right]=\left\langle\exp \left(i \sum_{E_{p}^{(R)}} J_{R}(z) X(z)\right)\right\rangle_{p} .
$$

We see that the integral over $X_{q}$ on the right-hand side is cancelled against that very integral in the denominator, leaving us with

$$
A_{p}\left[J_{R}\right] \sim \int D X_{R} \exp \left((2 \ln (p))^{-1} \sum_{E_{p}^{(R)}} X_{0}^{(R)} \widehat{\Delta}_{p} X_{0}^{(R)}+i \sum_{E_{p}^{(R)}} J_{R} X_{0}^{(R)}\right)
$$

From the definition of $X_{0}^{(R)}$,

$$
\hat{\Delta}_{p} X_{0}^{(R)}(z)=X_{0}^{(R)}\left(z_{1}\right)-X_{R}(z), \quad z \in E_{p}^{(R)},
$$

where $z_{1}$ is the unique vertex adjacent to $z$ which belongs to the sphere $E_{p}^{(R-1)}$.

In the limit $R \rightarrow \infty$ in (C.7),

$$
J_{R}(z) \rightarrow J(y)=i \sum_{j=1}^{N} k_{j} \delta\left(y, y_{j}\right),
$$

where $y \in \partial T_{p}$ and $\delta\left(y, y_{j}\right)$ is the delta function on $\partial T_{p}$. We shall assume that

$$
\lim _{R \rightarrow \infty} X_{0}^{(R)}(z)=X_{0}(y),
$$

a locally constant function on $\partial T_{p}$. [It is understood in (C.10) that $z \rightarrow y$ as $R \rightarrow \infty$.] Making use of the definition (3.17) of the normal derivative, and of Eq. (C.8), the 
limit is found to be

$$
\begin{aligned}
& A_{p}[J] \sim \int D X_{0} \exp \left(-\frac{p-1}{2 \ln (p)} \int_{\partial T_{p}} d \mu_{0}(y) X_{0}(y) \cdot \partial_{n}^{(p)} X_{0}(y)\right) \\
& \quad+i \int_{\partial T_{p}} d \mu_{0}(y) J(y) X_{0}(y) .
\end{aligned}
$$

For the derivative $\partial_{n}^{(p)} X_{0}$ an explicit expression follows from the Poisson formula on the tree (3.14):

$$
\partial_{n}^{(p)} X_{0}(y)=\left(1+p^{-1}\right)^{-1} \int_{\partial T_{p}} d \mu_{0}\left(y^{\prime}\right) \frac{X_{0}(y)-X_{0}\left(y^{\prime}\right)}{\left|y, y^{\prime}\right|_{p}^{2}} .
$$

Substituting this into (C.11) yields

$$
A_{p}[J] \sim \int D X_{0} \exp \left(-\tilde{S}_{p}\left[X_{0}\right]+i \int_{\partial T_{p}} d \mu_{0}(y) J(y) X_{0}(y)\right)
$$

with

$$
\widetilde{S}_{p}\left[X_{0}\right]=\frac{p(p-1)}{4(p+1) \ln (p)} \int_{\partial T_{p}} d \mu_{0}(y) d \mu_{0}\left(y^{\prime}\right) \frac{\left(X_{0}(y)-X_{0}\left(y^{\prime}\right)\right)^{2}}{\left|y, y^{\prime}\right|_{p}^{2}}
$$

This reproduces the non-local action of [13] after the identification $\partial T_{p} \cong Q_{p}$, and the subsequent reinterpretation of (C.14) as an integral over $Q_{p}[\operatorname{see}(2.10)$ and (4.19)]. The action (C.14) thus emerges as an "effective" action governing the field dynamics on the boundary of the world sheet.

Acknowledgements. I am grateful to L. O. Chekhov, A. A. Gerasimov, Ya. I. Kogan, D. R. Lebedev, A. S. Losev, A. V. Marshakov, A. D. Mironov, A. Yu. Morozov, J. Nekovar, A. M. Semikhatov, and B. L. Spokoiny for useful discussions at different stages of this work. I am especially grateful to Prof. Yu. I. Manin for his interest in the above results and useful suggestions. I also thank A. D. Mironov and A. M. Semikhatov for help in improving the manuscript.

\section{References}

1. Scherk, J.: An introduction to the theory of dual models and strings. Rev. Mod. Phys. 47, 123-164 (1975)

2. Polyakov, A.M.: Quantum geometry of bosonic strings. Phys. Lett. 103B, 207-210 (1981)

3. Beilinson, A.A., Manin, Yu.I.: The Mumford form and the Polyakov measure in string theory. Commun. Math. Phys. 107, 359-376 (1986)

4. Freund, P.G.O., Olson, M.: Non-archimedean strings. Phys. Lett. 199B, 186-190 (1987)

5. Volovich, I.V.: p-Adic string. Class. and Quant. Gravity 4, L83-L87 (1987);

Grossman, B.: p-Adic strings, the Weil conjectures and anomalies. Rockefeller University preprint DOE/ER/40325-7-Task B

6. Gervais, J.L.: $p$-Adic analyticity and Virasoro algebras for conformal theories in more than two dimensions. Phys. Lett. 201B, $306-310$ (1988)

7. Koblitz, N.: p-Adic numbers, p-adic analysis and zeta functions. Berlin, Heidelberg, New York: Springer 1977

8. Gelfand, I.M., Graev, M.I., Piateskii-Shapiro, I.I.: Representation theory and automorphic functions. Philadelphia, PA: Saunders 1969

9. Freund, P.G.O., Witten, E.: Adelic string amplitudes. Phys. Lett. 199B, 191-194 (1987)

10. Brekke, L., Freund, P.G.O., Olson, M., Witten, E.: Non-archimedean string dynamics. Nucl. Phys. B 302, 365-402 (1988) 
11. Brekke, L., Freund, P.G.O., Melzer, E., Olson, M.: Adelic $N$-point amplitudes. Chicago preprint EFI-88-34 (1988)

12. Zabrodin, A.V.: Non-archimedean string action and Bruhat-Tits trees. Mod. Phys. Lett. A 4, 367-374 (1989)

13. Knizhnik, V.G., Polyakov, A.M.: Unpublished (1987)

Parisi, G.: On p-adic functional integrals. Mod. Phys. Lett. A 3, 639-643 (1988);

Spokoiny, B.L.: Quantum geometry of non-archimedean particles and strings. Phys. Lett. 208 B, 401-406 (1988);

Zhang, R.B.: Lagrangian formulation of open and closed p-adic strings. Phys. Lett. 209B, 229-232 (1988)

14. Serre, J.P.: Trees. Berlin, Heidelberg, New York: Springer 1980

Manin, Yu.I.: p-Adic automorphic functions. Sovr. Probl. Mat. 3, 5-92. Moscow: VINITI 1974 (in Russian)

15. Bruhat, F., Tits, J.: Groupes reductifs sur un corps local. I. Publ. Math. IHES 41, 5-251 (1972)

16. Kubota, T.: Elementary theory of Eisenstein series. Tokyo: Kodansha Ltd. 1973

17. Jacquet, H., Langlands, R.P.: Automorphic forms on GL(2). Lecture Notes in Mathematics, Vol. 114. Berlin, Heidelberg, New York: Springer 1970

18. Helgason, S.: Groups and geometric analysis. Orlando: Academic Press 1984

19. Baxter, R.: Exactly solved models in statistical mechanics. London: Academic Press 1982

20. Frampton, P.H., Okada, Y.: $p$-Adic string $n$-point function. Phys. Rev. Lett. 60, 484-486(1988)

21. Tits, J.: Sur le groupe des automorphismes d'un arbre. In: Essays Topol. and Relat. Topics, pp. 188-211. Berlin, Heidelberg, New York: Springer 1970

22. Neretin, Yu.: Representations of Virasoro algebra and affine algebras. Sovr. Probl. Mat. 22, 163-224. Moscow: VINITI 1988 (in Russian)

23. Algebraic Number Theory. Cassels, J.W.S., Fröhlich, A. (eds.). London, New York: Academic Press 1967

24. Marshakov, A.V.: Zabrodin, A.V.: Work in progress

25. Cartier, P.: Harmonic analysis on trees. In: Harmonic analysis on homogeneous spaces. Proc. Symp. Pure Math., Vol. 26, pp. 419-424. Providence, R.I.: AMS 1973

Communicated by A. Jaffe

Received December 19, 1988 
